Submitted to Astrophysical Journal

\title{
Optical Spectroscopy of the environment of a ULX in NGC 7331
}

\author{
Pavel K. Abolmasov ${ }^{1}$, Douglas A. Swartz ${ }^{2}$, S. Fabrika ${ }^{1,3}$, Kajal K. Ghosh ${ }^{2}$, O. Sholukhova ${ }^{1}$, \\ and Allyn F. Tennant ${ }^{4}$
}

\begin{abstract}
Optical photometric and spectroscopic data are presented that show an association of an ultraluminous X-ray source in NGC 7331 with a young star cluster of mass $M=(1.1 \pm 0.2) \times 10^{5} \mathrm{M}_{\odot}$ and age $t_{c}=4.25 \pm 0.25 \mathrm{Myr}$. If the ULX is part of the bright stellar cluster, then the mass of the progenitor of the compact accretor must have been $\gtrsim 40-50 \mathrm{M}_{\odot}$ in order to already have evolved through the supernova stage to a compact object. The companion star is also likely an evolved massive star. The emission line spectrum of the nebula surrounding the cluster can be interpreted as a result of photoionization by the cluster OB stars with an additional source of shock excitation producing strong [S II], [O I] and $\mathrm{N}$ II lines. This additional source appears to be as much as five times more powerful than the supernovae and stellar winds in the cluster can provide. Additional mechanical energy input associated with the ULX itself can help explain the residual shock excited line luminosities of the emission region.
\end{abstract}

Subject headings: galaxies: individual (NGC 7331) — galaxies: star clusters — line: formation - X-rays: galaxies - X-rays: binaries - X-rays: individual (CXOU J223706)

\section{Introduction}

Ultraluminous X-ray sources (ULXs) are point-like non-nuclear sources in external galaxies with apparent X-ray luminosities greater than the Eddington limit of stellar mass

\footnotetext{
${ }^{1}$ Special Astrophysical Observatory, Nizhnij Arkhyz 369167, Russia

${ }^{2}$ Universities Space Research Association, NASA Marshall Space Flight Center, VP62, Huntsville, AL 35805 , USA

${ }^{3}$ University of Oulu, P.O. Box 3000, 90014, Finland

${ }^{4}$ Space Science Department, NASA Marshall Space Flight Center, VP62, Huntsville, AL, USA
} 
black holes, i.e., $L_{\mathrm{X}} \gtrsim 10^{39} \mathrm{ergs} \mathrm{s}^{-1}$. The existence of ULXs has been known since the first Einstein observations of nearby galaxies (Long \& van Speybroeck 1983; Helfand 1984; Fabbiano 1989) but only with the sub-arcsecond imaging capability of the Chandra X-ray Observatory have investigations at other wavelengths of the crowded fields of nearby galaxies become viable.

Multi-wavelength investigations enable a much broader range of diagnostic techniques in the study of ULXs than can be achieved by X-ray data analysis alone. In particular, traditional methods in optical imaging and spectroscopy can be used to constrain the age, metallicity and physical state of the local environments of ULXs. This, in turn, can help to determine the origin and history of the ULXs themselves and lead to a better understanding of the influence of ULXs on their surroundings.

Many of the models for ULXs favor young high-mass X-ray binary systems (see, e.g., the reviews by Fabbiano \& White (2006); Fabbiano (2006)). These objects should be preferentially associated with massive star forming regions such as OB associations and, at the high-mass limit, superstar clusters. These environments should be strong sources of line emission through photoionization by massive stars, supernovae (SNe) and by the ULX itself and may display enhanced forbidden line emission from shock excitation by stellar winds, SNe, and by energetic outflows and jets that could accompany the ULX X-ray emission. Nebulae such as those associated with the ULXs in Ho IX (Grisé et al. 2006a), NGC 5408 (Soria et al. 2005), NGC 5204 (Goad et al. 2002), and Ho II (Kaaret et al. 2004; Lehmann et al. 2005) are among the best-studied examples of this intricate ULX/star-formation connection.

As part of a program of spectroscopic studies of the local envrironments of ULX candidates, we have obtained a moderate-resolution long-slit spectrum of a ULX in NGC 7331. The X-ray characteristics of this ULX and the optical photometric properties of its environment are described in $\S$ 2. The ULX lies roughly in the center of an extended H II region, cataloged as P98 (Petit 1998), and within 30 pc of the center of a bright blue emission region. The optical spectrum of this emission region is presented in $\S$ 3. We model its spectrum as emission from a young star cluster combined with a nebula photoionized by a cluster hosting an X-ray source (§ 4). This model reproduces the observed $4000-7300 \AA$ continuum spectrum but under-predicts several strong observed emission lines. We argue ( $\S 4.3)$ these lines are due, at least in part, to shock excitation associated with the ULX and that radiative and mechanical feedback plays an important part in the overall energetics of the emission region. We briefly summarize our results and compare the properties of the environment of the ULX in NGC 7331 to other ULX nebulae in $\S$. 


\section{X-ray and Optical Observations}

NGC 7331 is a highly-inclined, $\mathrm{i}=77^{\circ}$ (García-Gómez et al. 2002), SA(s)b spiral galaxy subtending 10.'5 on the sky. We adopt a distance of $14.6 \mathrm{Mpc}$ (Ferrarese et al. 2000); 1" corresponds to $71 \mathrm{pc}$.

Swartz et al. (2004) identified three ULX candidates within the $D_{25}$ isophote of the Chandra field of NGC 7331. All three lie in the northeast portion of the spiral arm structure beyond the large ring seen in the Spitzer Infrared Nearby Galaxies Survey images (Regan et al. 2005). An $\mathrm{H} \alpha$ image of this region is shown in Figure 1 with the approximate positions of the three ULX candidates superposed. Only the brightest ULX candidate has an obvious association with optical emission features in this or any other images we have studied. For the remainder of this work, we will refer to this ULX, which is formally designated CXOU J223706.6+342620, as CXOU J223706.

\subsection{Chandra observations}

Chandra obtained a 29.5 ks observation of a portion of NGC 7331 on 12 Aug 2001 (ObsID 2198). The observation was taken in sub-array mode using the Advanced CCD Imaging Spectrometer (ACIS) with the galaxy center imaged near the aimpoint on the back-illuminated S3 CCD. CXOU J223706 is approximately 1.'85 off-axis in this observation. Using the known position of the nucleus 1 of NGC 7331, we estimate the absolute positional uncertainty of the ULX is $\sim 0^{\prime \prime}$. W. Within a $3 \sigma=1$ ".86 circle about the position of the source, $175 \mathrm{X}$-ray events were detected corresponding to a $\mathrm{S} / \mathrm{N}$ of 10.6 (in the $0.5-8.0 \mathrm{keV}$ band). Acceptable fits to the spectrum can be made using a variety of models. The best-fitting model attempted is an absorbed power law $\left(N_{H}=6.9_{-2.4}^{+3.1} \times 10^{21} \mathrm{~cm}^{-2}, \Gamma=2.26_{-0.52}^{+0.63}, \chi^{2}=5.1\right.$ for 5 dof after grouping to ensure at least 20 counts per spectral bin). The corresponding intrinsic source luminosity is $L_{\mathrm{X}}=(2.8 \pm 0.6) \times 10^{39} \mathrm{ergs} \mathrm{s}^{-1}$ according to our previous analysis (Swartz et al. 2004). (Here and elsewhere, errors are extremes on the single interesting parameter $1 \sigma$ confidence intervals.) The luminosity of CXOU J223706 lies at the median value of the 97 ULX candidates in our sample of ULXs in spiral galaxies. The average luminosity for that subsample is $5.9 \times 10^{39} \mathrm{ergs} \mathrm{s}^{-1}$ and the highest luminosities exceeded several $10^{40} \mathrm{ergs} \mathrm{s}^{-1}$. If we assume the X-ray luminosity approximates the bolometric luminosity and that the source radiates isotropically at the Eddington limit during the Chandra observation, then the mass of the compact accretor is $22 \pm 5 \mathrm{M}_{\odot}$.

$$
{ }^{1} \alpha=22^{h} 37^{m} 04^{s} .096, \delta=+34^{\circ} 24^{\prime} 56^{\prime \prime} .29 \text { (J2000); Argyle \& Clements (1990). }
$$




\subsection{Hubble observations}

Hubble/WFPC2 observations 2 of NGC7331 were carried out on 1997 August 13 and 14 using F450W and F814W filters. Astrometric corrections were made using USNO stars (Monet et al. 2003). The $1 \sigma$ nominal absolute positional uncertainities in the Hubble images are $0^{\prime \prime} .3$ giving a combined uncertainty of about 0.5 between the Hubble and Chandra registration.

Figure2 displays an $8^{\prime \prime} \times 8^{\prime \prime}$ portion of the F450W Hubble image around CXOU J223706. The Hubble image is dominated by two bright extended sources; presumably young stellar clusters. The X-ray source is located within the NW cluster, within the formal error. The optical source has a FWHM of $\sim 0^{\prime \prime} .1$ or 7 pc at the distance of NGC 7331 (the source is not completely symmetric, Hubble images reveal additional emission in the wing). The total background-corrected brightness within a 1".5 diameter circle (equivalent to the seeing conditions in the ground-based data, see below) centered on the X-ray source position is $m_{\mathrm{F} 450 \mathrm{~W}}=20.44 \mathrm{mag}$. This corresponds to an absolute magnitude $M_{450 \mathrm{~W}}=-10.4 \mathrm{mag}$, lying at about the maximal luminosity of the Galactic open clusters (Larsen 2001). However, taking into account interstellar absorption ( $(4)$, the object intrinsic brightness may be significantly higher.

\subsection{Isaac Newton Telescope observations}

Archival optical images of NGC 7331, obtained with the Wide Field Camera (WFC) on the Isaac Newton Telescope (INT) at La Palmas, were examined for structure local to CXOU J223706. We used images taken in narrow filters [O III] (central wavelength $5008 \AA$, FWHM=100 $\AA), \mathrm{H} \alpha(6568 \AA, 95 \AA)$ and in the Harris broad-band filters B (4298, $1065 \AA)$, $\mathrm{V}(5425 \AA, 975 \AA)$ and $\mathrm{R}(6380 \AA, 1520 \AA)$. All the imaging observations were carried out in 2000 and 2001. We have estimated seeing from the FWHM of unsaturated point-like sources in the images to be $0^{\prime \prime} .7$ in $\mathrm{H} \alpha, 0^{\prime \prime} .8$ in [O III] and from $0^{\prime \prime} .9$ to $1^{\prime \prime} .2$ in the broad bands.

Astrometric corrections were made using USNO-B1 and USNO-B2 standards (Monet et al. 2003). The astrometrical error obtained in all these images (using 22 to 27 astrometrical standard stars) is less than $0^{\prime \prime} 2$. We made photometric calibration of the B- and V-band images using 19 USNO standard stars that range in brightness from 13.0 to 20.5 mag. A systematic error of the USNO photometric standards as high as 0.2-0.3 magnitude is possible

\footnotetext{
${ }^{2}$ Available from http://archive.stsci.edu/

${ }^{3}$ Available from http://casu.ast.cam.ac.uk/casuadc/archives/ingarch
} 
for the fainter stars (Monet et al. 2003).

We created new $\mathrm{H} \alpha$ and [O III] images free of continuum emission by dividing the narrow band images over the $\mathrm{R}$ and $\mathrm{V}$ images, respectively. In the resulting images the majority of single stars (presumably non-emission-line stars) disappear. From these images one may estimate the spatial structure in the emission lines and measure the corresponding emission line equivalent widths. Isophotes at the 20,50 and $85 \%$ levels above the background in these $\mathrm{H} \alpha$ and [O III] images are shown in Figure 2 (100\% corresponds to the maximum value in the $8^{\prime \prime} \times 8^{\prime \prime}$ field). Taking the $50 \%$ isophote as representative of the extent of the emission in these lines, one obtains the size of the emission-line region to be $\sim 2^{\prime \prime}$ in $\mathrm{H} \alpha$ and [O III] corresponding to $140 \mathrm{pc}$ (FWHM). The peak of the $\mathrm{H} \alpha$ emission is coincident with the NW cluster resolved in the Hubble image. The peak [O III] emission lies between the two clusters suggesting each cluster contributes equally to the [O III] emission.

The estimated B-band magnitude within a circle of 1".5 diameter around the NW cluster is $19 \mathrm{~m} .97 \pm 0.06$ (USNO-B2) and $20 \cdot 32 \pm 0.06$ (USNO-B1). For modelling the optical spectrum (see below), we adopt the value $\mathrm{B}=20 \mathrm{~m} .0$.

\section{Optical Spectroscopy}

Optical spectral data were obtained using the $6 \mathrm{~m}$ Russian telescope with the SCORPIO focal reducer (Afanasiev \& Moiseev 2005) in the long-slit (LS) mode with a 0'.75-wide slit, providing spectral resolution of about $10 \AA$. The data cover the spectral range $4000-7300$ $\AA$. The object was observed on August 29, 2005 at 1".4 seeing conditions. The reduction process includes all the standard procedures.

The spectrum is shown in Figure 3. Many strong emission lines are present above a well-defined blue continuum. Table 1 lists the observed and dereddened integral fluxes in all the emission lines detected.

We extracted slit images in the strongest lines $(\mathrm{H} \beta,[\mathrm{O} \mathrm{III}], \mathrm{H} \alpha$, and $[\mathrm{S} \mathrm{II}])$. The extent of the [O III] and $\mathrm{H} \alpha$ emission in the slit images is consistent with the structure seen in the INT images. The emission in the slit images can best be described as the sum of a point-like structure (FWHM 1".5 consistent with the seeing conditions) and more extended (FWHM 5".0) emission. That is, the two clusters are not resolved. The centroids of the point-like emission in all the lines are coincident within their errors; but in the extended component the [O III] centroid is shifted about 0.4 to the SSE relative to $\mathrm{H} \alpha$ along the slit (cf. Figure 2). The [S II] centroid is shifted in the opposite direction, about 0.2 to the NNW relative to the $\mathrm{H} \alpha$ peak. 


\section{Spectrum modelling}

The size and luminosity of the bright sources in the Hubble blue (F450W) image suggest they are compact young star clusters. The INT narrow-band images and SAO slit images suggest the star clusters are embedded in a larger emission line nebula (with the slit sampling most of the cluster emission but not all of the larger emission line region). We constructed synthetic spectra based on a model for the emission consisting of an underlying young stellar population and a photoionized nebula. Starburst99 (Vázquez \& Leitherer 2005) was used for the stellar contribution which provides model spectra for the continuum emission as functions of age and metallicity of the cluster and normalized by the cluster mass. CLOUDY96.01 (Ferland et al. 1998) was used to simulate the emission line spectrum from the photoionized nebula as a function of the input photoionizing source spectrum and ionization parameter, the nebular abundances, and the density. The spectral modelling began by finding the bestfitting cluster model. This model was then subtracted from the observed spectrum and the best-fitting model for the residual spectrum was found using the photoionization code.

\subsection{The contribution from the Star Cluster}

The oxygen abundance and abundance gradient in NGC 7331 is given by Pilyugin et al. (2003). At the location of CXOU J223706, about 90" from the center of the galaxy, $12+$ $\log (\mathrm{O} / \mathrm{H})=8.32$ or about 2-3 times less than the solar value. Using this as a guide, we constructed Starburst99 models with $[\mathrm{Fe} / \mathrm{H}]=0.0,-0.4,-0.7$. We computed a grid of models for cluster ages, $t_{c}$, ranging from 1 to 30 Myr. The model spectra were fit to the observed spectra allowing for a variable interstellar absorption component (using the reddening curve of Cardelli et al. (1998)). Since the Starburst99 stellar cluster model does not predict a nebular emission line spectrum it was necessary to exclude all the bright observed emission lines in the model fitting. We find the best-fit parameters of the cluster model are $t_{c}=(4.25 \pm 0.25) \mathrm{Myr}, A_{V}=1.43 \pm 0.05, M=(1.1 \pm 0.2) \times 10^{5} \mathrm{M}_{\odot}$ where $M$ is the cluster mass which scales linearly with the overall model normalization. The $\chi_{r}^{2}$ statistic for this model is 1.30 for $\sim 200$ degrees of freedom (the spectral range divided by the spectral resolution element with emission lines omitted and reduced by the number of model fit parameters). Solar metallicity provides a significantly better fit. The best-fit model spectrum (scaled by a factor of $1 / 3$ for clarity) is shown as the lower curve in Figure 3 ,

The age and metallicity derived here are consistent with the presence of the broad but weak emission features observed at $4650 \AA$ and $5806 \AA$. We interpret these features as the $\mathrm{C}$ III+N III and $\mathrm{C}$ IV line blends, respectively, due to the presence of Wolf-Rayet stars (Conti et al. 1983; Schaerer \& Vacca 1998) in the cluster. We estimate the equivalent 
widths to be $1.7 \pm 0.2 \AA$ for the blue blend and $4.2 \pm 0.2 \AA$ for the red blend of lines. (The quoted errors are formal errors using a piece-wise linear approximation to the local continua; inspection of Figure 3 suggests the uncertainties are likely higher.) The theoretical blue-tored ratio (Schaerer \& Vacca 1998) most closely approaches the observed ratio at 3-6 Myr where the theoretical ratio is $\approx 1$ for a wide range of metallicities.

\subsection{The contribution from photoionization}

The best-fit stellar population model spectrum was subtracted from the dereddened observed spectrum to give an estimate of the purely nebular spectrum. We used the $3 \leq$ $t_{c} \leq 6 \mathrm{Myr}$ UV spectrum from the best-fit unabsorbed cluster model plus an additional X-ray component based on the Chandra spectrum of CXOU J223706 $\left(\Gamma=2.2,3.2 \times 10^{48}\right.$ photons $\mathrm{s}^{-1}$ blueward of the Ly edge) for the photoionization source for the nebula model. We calculated a set of spherically-symmetric photoionization models with fixed inner $(1 \mathrm{pc})$ and outer (30 pc) radii using CLOUDY96.01. The inner radius is typical for a massive young star cluster (e.g., Nilakshi et al. (2002)). The outer radius corresponds to the half-width of the slit in projection (at the galaxy distance of $14.6 \mathrm{Mpc}$ ). We have found that the choice of inner and outer radii do not strongly affect the results. The nebula gas density and the low-energy cutoff for the ionizing X-ray spectrum were allowed to vary in the modelling. The bestfitting gas density is about $30 \mathrm{~cm}^{-3}$ (consistent with the observed [S II] $\lambda 6717 / \lambda 6731$ ratio). The models proved insensitive to the additional X-ray and corresponding EUV radiation from the ULX. This was anticipated because the number flux from the cluster model in the Ly continuum $\left(\sim 10^{51} \mathrm{~s}^{-1}\right)$ far exceeds the flux of X-ray photons even after extrapolating this rather steep X-ray spectrum to the Ly edge.

The results of the photoionization model predictions and the observed emission line parameters are listed in Table 1 . For each emission line observed, the ratio of the observed (Column 2) and dereddened (Column 3; $A_{V}=1{ }^{\mathrm{m}} \cdot 4$ ) line fluxes to the $\mathrm{H} \beta$ flux are tabulated along with the integral dereddened line luminosity (Column 4), the photoionization model predicted line luminosity (Column 5), and the residual line luminosity after subtracting the photoionization model prediction (Column 6). The best-fit stellar continuum model combined with the best-fit photoionization model (convolved with the instrumental response function) is shown as the middle (dotted) curve in Figure 3 (displaced by $1 / 2$ for clarity). 


\subsection{The contribution from shocks}

These pure photoionization models predict strong [O III], He I, and $\mathrm{H}$ Balmer line emission excited by the UV emission from the underlying star cluster but are unable to reproduce the observed strength of several key emission lines (see Table 1 and Figure 3). A significant excess is observed in [S II] $\lambda \lambda 6716,6731,[\mathrm{OI}] \lambda \lambda 6300,6364$ and [NII] $\lambda \lambda 6548$, $6583 \AA$ doublets. We find the additional dereddened luminosity in the $[\mathrm{S} \mathrm{II}]$ doublet, for example, is $L_{[\mathrm{SII}]}=1.72 \times 10^{38} \mathrm{ergs} \mathrm{s}^{-1}$. Further, the residual [O III] $\lambda(4959+5007) / \lambda 4363$ ratio is low, $\sim 20$, compared to typical photoionized H II regions.

The observed excesses in these low-excitation lines and the low [O III] ratio are likely caused by shock excitation in the nebula (cf. Dopita et al. (2000)). We can identify three plausible sources for shock excitation: ( $i$ ) individual SNe in the stellar cluster, $(i i)$ the cumulative stellar wind in the cluster, and (iii) the ULX source. Here we make only rough estimates of the possible contributions from these three sources; we lack sufficient knowledge of the physical conditions in the nebula (e.g., shock velocity, pressure, density, temperature, and pre-shock ionization) to apply a full shock-excitation model for the emission lines.

The Starburst99 models predict the total supernova production rate in a cluster of age $t_{c} \sim 4.25 \mathrm{Myr}$ and mass $M \sim 10^{5} \mathrm{M}_{\odot}$ and a Salpeter IMF is about $6 \times 10^{-5} \mathrm{yr}^{-1}$. Since SNRs are visible in emission lines for up to $10^{5}$ years (Cioffi et al. 1988), the effective number of SNRs contributing to the spectrum is at most $\sim 6$. Typical $\mathrm{H} \alpha$ and $[\mathrm{S}$ II] luminosities from an individual SNR are $L_{\mathrm{H} \alpha} \sim L_{[\mathrm{SII}]} \sim(3-6) \times 10^{36} \mathrm{ergs} \mathrm{s}^{-1}$ (Braun \& Walterbos 1993; Matonick \& Fesen 1997). The expected luminosity in these lines from 6 SNRs is then $\sim(1.8-3.6) \times 10^{37} \mathrm{ergs} \mathrm{s}^{-1}$, or 5 to 10 times less than the residual luminosities listed in Table 1 .

At the estimated age of 4.25 Myr, the Starburst99 model predicts equal contributions from SNe and stellar winds to the mechanical luminosity of the cluster. If the conversion efficiencies from mechanical luminosity input to emission line radiation are the same for SNe and stellar winds, winds will contribute a comparable amount to the shock-excited lines. The combination of SNe and stellar winds still leaves the [S II] $\lambda \lambda 6717,6731$ production about 2.5 to 5 times below that needed to explain the observed luminosity.

The third plausible source for shock excitation is the ULX. The presence of such an object in the cluster may contribute to the residual line emissions if mechanical energy from the X-ray source can be imparted to its surroundings in the form of an outflow. It has been postulated that some ULXs are microquasars (King et al. 2001), whose radiation is collimated along the jet axis, or supercritical accretion disks with outflows like that in SS433 (Fabrika \& Mescheryakov 2001). 
In the case of microquasars, outflow in the form of a leptonic jet could occur during the low state and during outbursts associated with transitions within the very-high/intermediate states (Fender, Pooley, \& Gallo 2004; Corbel et al. 2003). However, utilizing the known correlation between X-ray and radio luminosities for black holes in the low-hard X-ray state (Corbel et al. 2003; Gallo, Fender, \& Pooley 2003) to estimate the mechanical power in the jet and assuming the Chandra observation of CXOU J223706 was obtained when the source was near Eddington, then the power in the jet is $L_{J} \lesssim 10^{38} \mathrm{ergs} \mathrm{s}^{-1}$ even allowing for additional transient ejection events (Fender, Belloni, \& Gallo 2004). The mechanical power imparted to the surrounding nebula in the form of outflow from microquasar-like jets from the ULX is then far less than needed to explain the residual line emission. [Note that the Galactic microquasars like 1E 1740.7-2942 and GRS 1758-258 (Mirabel et al. 1993), for

instance, have presistent lobe-like emission but are old stellar systems lacking associations with young energetic nebulae.]

In the case of SS433-like objects, outflow is in the form of persisent heavy jets that impart a large fraction of their accretion energy into their surroundings in the form of mechanical energy. The observed kinetic luminosity in the two jets of SS433 is $\sim 2 \times 10^{39} \mathrm{ergs} \mathrm{s}^{-1}$ (Fabrika 2004). This is comparable to the predicted mechanical luminosity from stellar winds and SNe in the star cluster associated with CXOU J223706, 3.5 × $10^{39} \mathrm{ergs} \mathrm{s}^{-1}$, at its age of $4.25 \mathrm{Myr}$.

Likely all three sources of shock excitation contribute to the observed line emission. The ULX can be an important, and even dominant, source of radiative and mechanical feedback in the overall energetics of the emission region.

\section{Discussion}

We have presented optical photometric and spectroscopic data that help to describe the physical environment surrounding the ULX CXOU J223706 in NGC 7331. CXOU J223706 is spatially-coincident (within astrometrical errors) with a bright, young, $t_{c}=4.25 \pm 0.25 \mathrm{Myr}$, star cluster of mass $M=(1.1 \pm 0.2) \times 10^{5} \mathrm{M}_{\odot}$. If the ULX is a part of this bright stellar cluster, then the progenitor of the compact accretor must have been at least as massive as 40-50 $\mathrm{M}_{\odot}$ in order to have already evolved through the supernova stage. The companion star is also likely massive and evolved in order to provide the high mass accretion rate needed to power the ULX.

The emission line spectrum of the surrounding nebula can be interpreted as a result of photoinization produced by the cluster UV spectrum, but an additional source of shock 
excitation is needed to understand the observed [S II], [OI] and [NII] line intensities. The mechanical input from SNe and stellar winds from the star cluster can account for only $\sim 20-40 \%$ of the required shock excitation so some additional energy source is needed. The obvious candidate is the ULX itself.

Emission nebulae associated with nearby ULXs are apparently a common phenomenon. Although no formal census has been undertaken, we find potential associations with bright, extended optical structures for 67 of the 154 ULX candidates tabulated in the ULX survey of Swartz et al. (2004). Although these associations are based mainly upon Digitized Sky Survey images and must await more conclusive analysis, many detailed studies of ULXs with associated nebulae have appeared in the recent literature (Pakull \& Mirioni 2003; Lehmann et al. 2005; Kuntz et al. 2005; Pakufl et al. 2006; Grisé et al. 2006a.,b; Abolmasov et al. 2007).

In particular, the nebula associated with CXOU J223706 in NGC 7331 can be compared with the compact nebula MF 16 associated with the ULX NGC 6946 X-1 (Blair et al. 2001). The MF 16 nebula is an isolated object: It does not appear to be contaminated by associated star clusters or nearby H II regions. The line luminosities of MF 16 (Blair et al. 2001; Abolmasov et al. 2006) are $L_{[\mathrm{SII}]} \approx L_{\mathrm{H} \alpha} \approx 2 \times 10^{38} \mathrm{ergs} \mathrm{s}^{-1}, L_{\mathrm{H} \beta} \approx 6 \times 10^{37} \mathrm{ergs} \mathrm{s}^{-1}$; equal to the residual line luminosities in the NGC 7331 nebula (Table 1). On the other hand, [O III] $\lambda \lambda 4959,5007$ and [O I] $\lambda 6300 \AA$ lines in MF 16 are 3.5 times brighter than those in the NGC 7331 residuals. These two lines are excited in different ways: by UV photoionization in the case of [O III] $\lambda \lambda 4959,5007$ and by shock excitation in the case of [O I] $\lambda 6300 \AA$.

Additional sources of mechanical energy in the form of more or less collimated winds or jets is not unprecedented in ULX nebulae. In the nebulae of ULXs NGC 6946 X-1 and Holmberg II X-1 (another "isolated" ULX nebula), radial velocity gradients of 50-100 km s" have been found (Lehmann et al. 2005; Fabrika et al. 2006). It was concluded that the nebulae have to be powered by the central ULXs and that, therefore, the ULXs must also provide a source of gas collisional excitation in the ULX-associated nebulae. It was found recently through optical spectroscopy of 8 ULX-associated nebulae (Abolmasov et al. 2007) that in at least half of them shock excitation dominates. We cannot say definitely about the kinematical properties of the gas in the CXOU J223706 counterpart - we lack both spectral and spatial resolution for this - but it is likely the ULX plays an important role here as well.

The authors thank N. Borisov for help with the observations. This work has been supported in part by Russian RFBR grants NN 06-02-16865 and 07-02-00909 and by a joint RFBR/JSPS grant N 05-02-12710. Additional support was provided by NASA under grant NNG04GC86G issued through the Office of Space Science and by the Space Telescope Science Institute under the grant HST/AR-10954. 


\section{REFERENCES}

Abolmasov, P., Fabrika, S., Sholukhova, O. \& Afanasiev, V. 2005, in Science Perspectives for 3D Spectroscopy, ed. M. Kissler-Patig, M. M. Roth. \& J. R. Walsh (Springer Berlin / Heidelberg); astro-ph/0602369

Abolmasov, P., Fabrika, S., Sholukhova, O. \& Afanasiev, V. 2007, Astrophysical Bulletin, 62, N 1, 36; astro-ph/0602369

Afanasiev, V.\& Moiseev, A. 2005, Astronomy Letters, 31, 194

Argyle, R. W. \& Clements, E. D. 1990, The Observatory, 110, 93

Blair, W. P., Fesen, R. A. \& Schlegel, E. M. 2001, AJ, 121, 1497

Braun, R. \& Walterbos, R. A. M. 1993, A\&ASS, 98, 327

Cardelli, J. A., Clayton, G. C. \& Mathis, J. S. 1998 ApJ, 345, 245

Cioffi, D. F., McKee, C. F. \& Bertschinger, E. 1988 ApJ, 334, 252

Conti, P. S., Leep, M. E. \& Perry, D. N. 1983 ApJ, 268, 228

Dopita, M. A., Kewley, L. J., Heisler, C. A. \& Sutherland, R. S. 2000 ApJ, 542, 224

Dopita, M. A. \& Sutherland, R. S. 1996, ApJSS, 102, 161

Fabbiano, G. 1989, ARA \& A, 27, 87

Fabbiano, G. 2006, ARA\&A, 44, 323

Fabbiano, G. \& White, N. E. 2006, in Compact stellar X-ray sources, ed. W. Lewin \& M. van der Klis, Cambridge Astrophysics Series No. 39, (Cambridge:Cambridge University Press), 475

Fabrika, S., Mescheryakov A. 2001. In: Galaxies and their Constituents at the Highest Angular Resolution, IAU Symp. N205, ed. R.T. Schilizzi, p. 268

Fabrika, S., 2004, Astrophys. and Space Phys. Rev., 12, 1

Fabrika, S., Karpov, S., Abolmasov, P. \& Sholukhova, O. 2006. In Populations of High Energy Sources in Galaxies, IAU Symposium 230, ed. E. J. A. Meurs \& G. Fabbiano (Cambridge : Cambridge University Press); astro-ph/0510491

Fender, R. P., Belloni, T. P. \& Gallo, E. 2004, MNRAS, 355, 1105 
Ferland, G. J., Korista, K. T., Verner, D. ., Ferguson, J. W., Kingdon, J. B. \& Verner, E. M. 1998, PASP, 110, 761

Ferrarese, L., et al. 2000, ApJ, 529, 745

Gallo, E., Fender, R. P. \& Pooley, G. G. 2003, MNRAS, 344, 60

García-Gómez, C., Athanassoula, E. \& Barber, C. 2002, A \& A, 389, 68

Goad, M. R., Roberts, T. P., Knigge, C., \& Lira, P. 2002, MNRAS, 335, 67

Grisé, F., Pakull, M. W. \& Motch, C. 2006, in Populations of High Energy Sources in Galaxies, IAU Symposium 230, ed. E. J. A. Meurs \& G. Fabbiano (Cambridge : Cambridge University Press); astro-ph/0603768

Grisé, F., Pakull, M. W. \& Motch, C. 2006, in Populations of High Energy Sources in Galaxies, IAU Symposium 230, ed. E. J. A. Meurs \& G. Fabbiano (Cambridge : Cambridge University Press)

Helfand, D. J. 1984, PASP, 96, 913

Kaaret, P., Ward, M. J., \& Zezas, A. 2004, MNRAS, 351, 83

King, A. R., Davies, M. B., Ward, M. J., Fabbiano, G., Elvis, M. 2001, ApJ (Letters), 552, L109 (2001)

Kuntz, K. D., Gruendl, R. A. \& Chu, Y. -H. et al. 2005 ApJ, 620, 31

Larsen, S. S. 2001, in Extragalactic Star Clusters, IAU Symposium 207, ed. D. Geisler, E. K. Grebel \& D. Minniti (Astronomical Society of the Pacific, San Francisco), 421

Lehmann, I., et al. 2005, A\& A, 431, 847

Leitherer, C., et al. 1999, ApJSS, 123, 3

Long, K. S. \& van Speybroeck, L. P. 1983, Accretion Driven Stellar X-ray Sources, ed. W. H.G. Lewin, \& E. P.J. van den Heuvel (Cambridge: Cambridge University Press), 141

Matonick, D. M., Fesen, R. A., 1997 ApJSS, 112, 49

Mirabel, I. F., Rodriguez, L. F., Cordier, B., Paul, J. \& Lebrun, F. 1993, A\& A SS, 97, 193

Monet. D. G., et al. 2003, AJ 125, 984

Nilakshi, S. R., Pandey, A. K. \& Mohan, V. 2002, A\&A, 383, 153 
Pakull, M. W. \& Mirioni, L. 2003, in Winds, Bubbles, and Explosions: a conference to honor John Dyson, RevMexAA (Serie de Conferencias) 15, 197, ed. J. Arthur \& W. J. Henney

Pakull, M. W., Grisé, F., Motch, C. 2006, in Populations of High Energy Sources in Galaxies, IAU Symposium 230, ed. E. J. A. Meurs \& G. Fabbiano (Cambridge : Cambridge University Press); astro-ph/0603771

Pilyugin, L. S., Thuan, L. X. \& Vílchez, J. M. 2003, A\&A, 397, 487

Petit, H. 1998, A\&AS, 131, 317

Regan, M. W., et al. 2004 ApJSS, 154, 204

Schaerer, D. \& Vacca, W. D. 1998, ApJ, 497, 618

Soria, R., Fender, R. P., Hannikainen, D. C., Read, A. M. \& Stevens, I. R. 2006, MNRAS, 368,1527

Swartz, D. A., Ghosh, K. K., Tennant, A. F. \& Wu, K. 2004 ApJS, 154, 519

Vázquez, G. A. \& Leitherer, C. 2005, ApJ, 621, 695 
Table 1: Integrated Emission Line Fluxes and Luminosities

\begin{tabular}{lccccc}
\hline \hline line & $F / F\left(H_{\beta}\right)$ & $F / F\left(H_{\beta}\right)$ & $L$ & $L_{\text {mod }}$ & $L_{\text {res }}$ \\
\cline { 4 - 6 } & & $($ dereddened $)$ & \multicolumn{2}{c}{$10^{37}$} & $\mathrm{ergs} \mathrm{s}^{-1}$ \\
\hline $\mathrm{H} \delta$ & $0.05 \pm 0.07$ & $0.211 \pm 0.012$ & $4.5 \pm 0.3$ & 3.8 & 0.7 \\
$\mathrm{H} \gamma$ & $0.34 \pm 0.02$ & $0.444 \pm 0.016$ & $9.5 \pm 0.5$ & 6.8 & 2.7 \\
{$[\mathrm{OIII}] \lambda 4363$} & $0.03 \pm 0.01$ & $0.04 \pm 0.02$ & $0.87 \pm 0.6$ & 0.0007 & 0.87 \\
$\mathrm{H} \beta$ & $1.000 \pm 0.05$ & $1.000 \pm 0.006$ & $21.45 \pm 0.19$ & 14.59 & 6.86 \\
{$[\mathrm{OIII}] \lambda 4959$} & $0.35 \pm 0.04$ & $0.292 \pm 0.008$ & $6.28 \pm 0.17$ & 2.32 & 3.96 \\
[OIII] $\lambda 5007$ & $1.15 \pm 0.05$ & $0.919 \pm 0.006$ & $19.72 \pm 0.19$ & 6.98 & 12.74 \\
{$[\mathrm{NI}] \lambda 5200$} & $0.08 \pm 0.04$ & $0.050 \pm 0.006$ & $1.08 \pm 0.17$ & 0.004 & 1.08 \\
{$[\mathrm{NII}] \lambda 5755$} & $0.02 \pm 0.03$ & $0.011 \pm 0.005$ & $0.24 \pm 0.11$ & 0.006 & 0.24 \\
$\mathrm{HeI} \lambda 5876$ & $0.13 \pm 0.04$ & $0.085 \pm 0.005$ & $1.81 \pm 0.14$ & 2.01 & -0.19 \\
{$[\mathrm{OI}] \lambda 6300+[\mathrm{SIII}] \lambda 6310$} & $0.15 \pm 0.04$ & $0.085 \pm 0.007$ & $1.83 \pm 0.15$ & 0.01 & 1.81 \\
{$[\mathrm{OI}] \lambda 6363$} & $0.02 \pm 0.02$ & $0.008 \pm 0.003$ & $0.18 \pm 0.09$ & 0.004 & 0.18 \\
{$[\mathrm{NII}] \lambda 6548$} & $0.668 \pm 0.003$ & $0.349 \pm 0.002$ & $7.48 \pm 0.05$ & 0.33 & 7.15 \\
$\mathrm{H} \alpha$ & $6.375 \pm 0.009$ & $3.360 \pm 0.005$ & $72.09 \pm 0.16$ & 44.77 & 27.32 \\
{$[\mathrm{NII}] \lambda 6583$} & $2.00 \pm 0.009$ & $1.046 \pm 0.005$ & $22.43 \pm 0.15$ & 0.97 & 21.46 \\
$\mathrm{HeI} \lambda 6678$ & $0.04 \pm 0.03$ & $0.023 \pm 0.004$ & $0.50 \pm 0.11$ & 0.57 & -0.07 \\
{$[\mathrm{SII}] \lambda 6717$} & $0.97 \pm 0.02$ & $0.493 \pm 0.011$ & $10.5 \pm 0.3$ & 0.6 & 10.0 \\
{$[\mathrm{SII}] \lambda 6731$} & $0.71 \pm 0.02$ & $0.356 \pm 0.011$ & $7.6 \pm 0.3$ & 0.4 & 7.2 \\
\hline
\end{tabular}




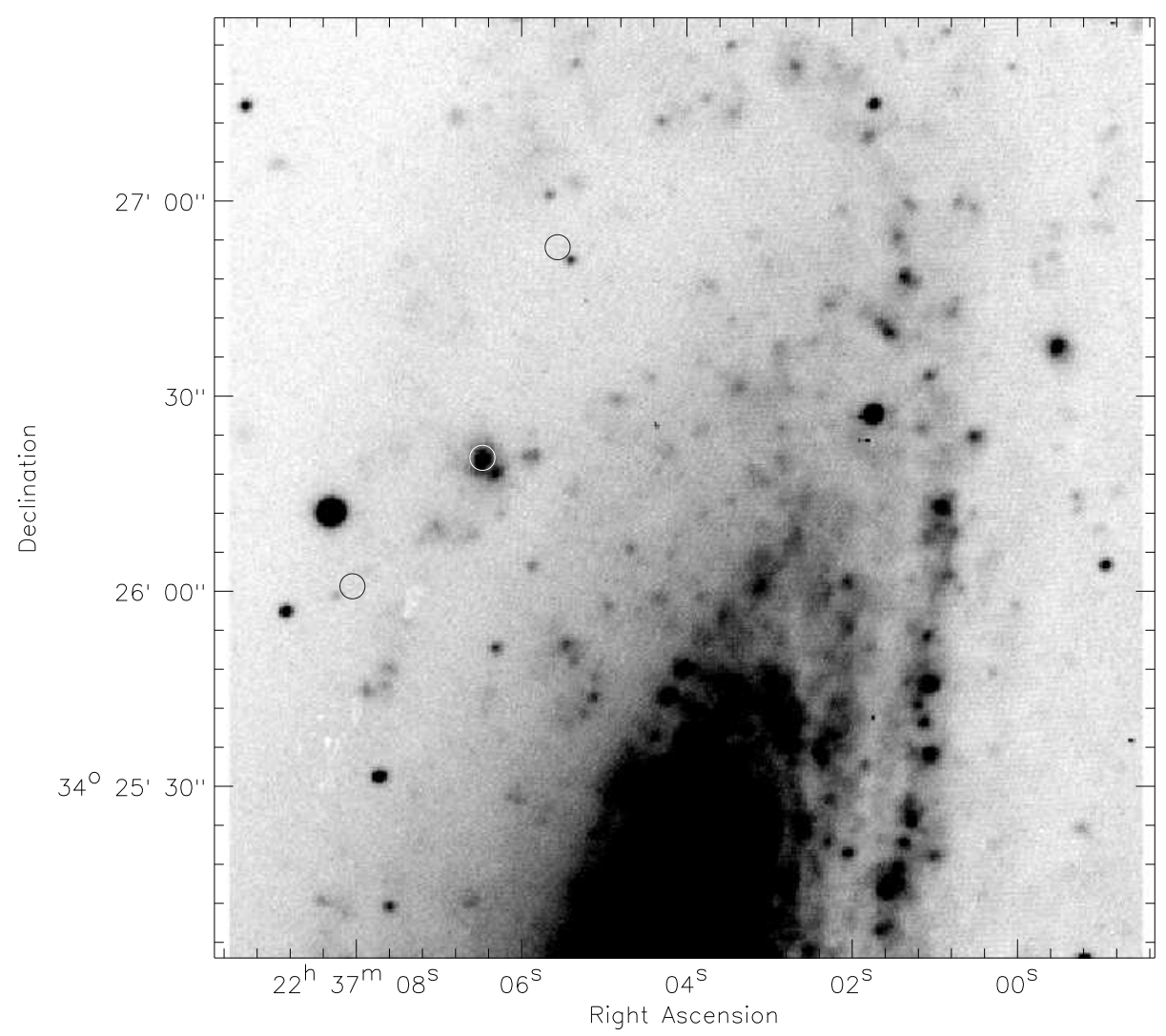

Fig. 1. - Isaac Newton Telescope $\mathrm{H} \alpha$ image of a portion of the NGC 7331 field showing the location of three ULXs in relation to the central regions of the galaxy. At the distance of 14.6 Mpc to NGC 7331, $1^{\prime}$ corresponds to $4.25 \mathrm{kpc}$. Circles denote $2^{\prime \prime}$ radius locations of the three ULX candidates detected. CXOU J223706 is the middle source. 


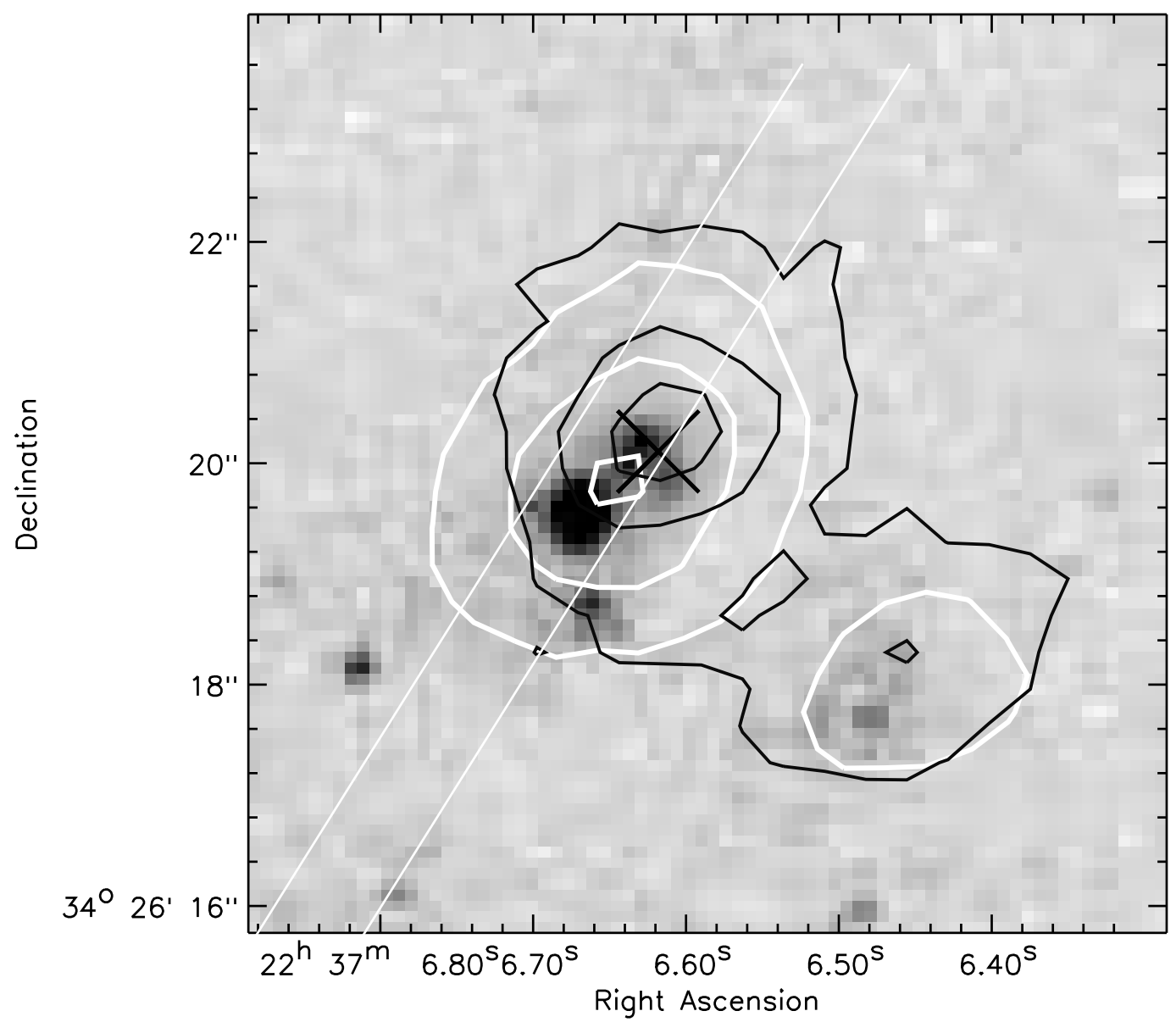

Fig. 2.- $8^{\prime \prime} \times 8^{\prime \prime}$ Hubble F450W image of the field around the ULX source CXOU J223706. The X-ray source position is marked by the " $\mathrm{X}$ " of \pm 0 " 5 radius corresponding to the combined Chandra and Hubble positional uncertainty. Ground-based $\mathrm{H} \alpha$ (black) and [O III] $\lambda 5007$ (white) isophotes at the 20,50 and $85 \%$ levels above their backgrounds are superimposed (see $\S 2.3)$. The slit position of the optical spectrum $(\S[3)$ is also shown. 


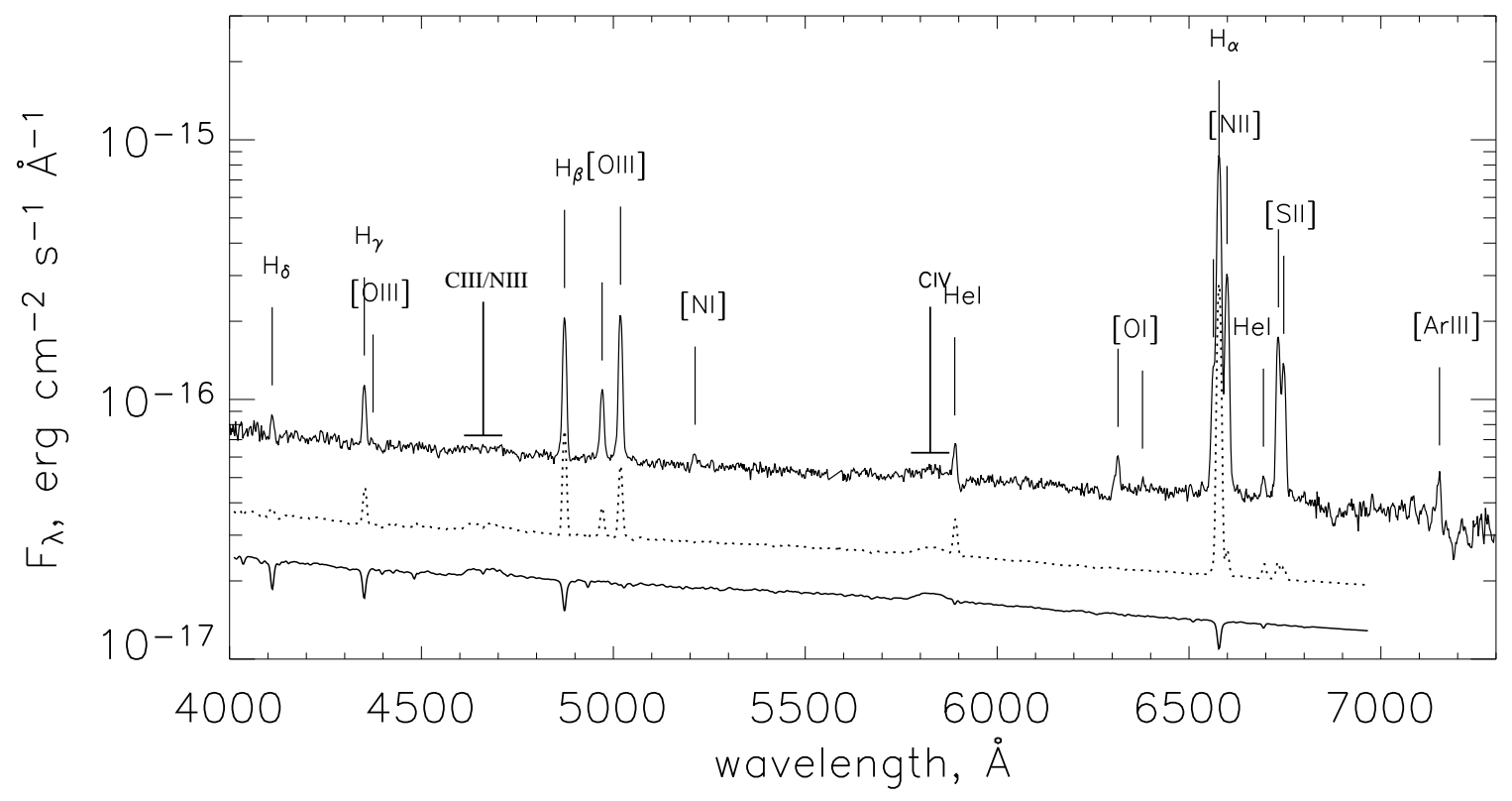

Fig. 3.- Integral spectrum of the cluster near CXOU J223706 (top). The lower thick solid curve is the best-fitting stellar spectrum model estimate, divided by a factor of 3 for clarity, and the dotted curve is the combination of this model and the best-fitting photoionization model (see $\S$ 4), divided by a factor of 2 . The brightest emission lines including Wolf-Rayet blends are superscribed. 